\title{
Sparse inverse and characteristic polynomial of generalized arrow matrix
}

\author{
Murray Dow*
}

(Received 12 September 1997; revised 16 December 1997)

\begin{abstract}
A generalized arrow matrix of order $n$ with $m$ non-zero rows and columns is presented. If a simple condition holds, the inverse of this matrix is also an arrow matrix of the same form. We then derive a simple expression for its characteristic polynomial.
\end{abstract}

*Supercomputer Facility, Australian National University, Canberra 0200, AustraliA, mailto:m.dow@anu.edu.au

See http://jamsb.sci.usq.edu.au/V39/E008/home.html for this paper and ancillary services, (c) Austral. Mathematical Soc. 1998. Published 20 March 1998, last corrected March 20, 1998. 


\section{Contents}

1 Introduction

668

2 Inverse of an arrow matrix

669

3 Characteristic Polynomial

672

References

676

\section{Introduction}

When testing numerical routines, a library of matrices with known inverses or other properties is useful. Most matrices with known inverses have none or at most two parameters that can be varied to provide a family of tests [3, 8]. More complex examples can be generated with the Sherman-Morrison formula or by Schur complements [2], but there appear to be very few simple test matrices of general size with many parameters. Notable exceptions to this are the Vandermonde and some tri-diagonal matrices $[1,3]$. The arrow matrix $[4,6,7]$ is another example; it is not hard to derive simple expressions for the inverse, determinant and characteristic polynomial of the arrow matrix which has the last row and column non-zero, and a non-zero diagonal [9].

We give a generalization of the arrow matrix, with an arbitrary number of 
non-zero columns and rows, whose inverse is also an arrow matrix, and we also give a simple expression for its characteristic polynomial. Such matrices and methods are also of interest as pre-conditioners for iterative processes, because of the sparsity of either the matrix or the inverse, and the freedom in choosing many of the elements.

\section{Inverse of an arrow matrix}

Definition 1 Let $M$ be the arrow matrix of order $n$

$$
M=\left[\begin{array}{ll}
D & e^{\prime} \\
f & A
\end{array}\right]
$$

where $D$ is a diagonal matrix order $n-m, A$ is square of order $m$, e and $f$ are $m \times(n-m)$ with constant rows, i.e.

$$
e=\left[\begin{array}{cccc}
e_{1} & e_{1} & \cdots & e_{1} \\
e_{2} & e_{2} & \cdots & e_{2} \\
\vdots & \vdots & & \vdots \\
e_{m} & e_{m} & \cdots & e_{m}
\end{array}\right], \quad f=\left[\begin{array}{cccc}
f_{1} & f_{1} & \cdots & f_{1} \\
f_{2} & f_{2} & \cdots & f_{2} \\
\vdots & \vdots & & \vdots \\
f_{m} & f_{m} & \cdots & f_{m}
\end{array}\right] .
$$

In general the inverse of $M$ will be dense, however provided $D$ is diagonal and a simple condition is satisfied, $M^{-1}$ will be sparse, and indeed will be another 
arrow matrix. Let the inverse of $M$ be

$$
M^{-1}=\left[\begin{array}{cc}
C & p^{\prime} \\
q & B
\end{array}\right] .
$$

Then using the usual formulae we obtain (see [5]) $C=\left(D-e^{\prime} A^{-1} f\right)^{-1}$. Now for $M^{-1}$ to be an arrow matrix, we require that $C$ be diagonal. This implies that $D$ be diagonal and that $e^{\prime} A^{-1} f=0$.

Theorem 1 Assuming that $A^{-1}$ exists, the inverse of the arrow matrix $M$ is given by

$$
M^{-1}=\left[\begin{array}{cc}
D^{-1} & p^{\prime} \\
q & B
\end{array}\right],
$$

where

$$
\begin{aligned}
q & =-A^{-1} f D^{-1}, \\
p^{\prime} & =-D^{-1} e^{\prime} A^{-1}, \\
B & =A^{-1}+q D p^{\prime},
\end{aligned}
$$

provided

$$
e^{\prime} A^{-1} f=0 .
$$

Writing the inverse as a matrix of cofactors, this condition can be transformed into

$$
\left|\begin{array}{cc}
0 & \bar{e}^{\prime} \\
\bar{f} & A
\end{array}\right|=0,
$$


where $|D|$ denotes a determinant and by $\bar{f}$ we mean the first column of $f$, similarly for $\bar{e}$ :

$$
\bar{e}=\left[\begin{array}{c}
e_{1} \\
e_{2} \\
\vdots \\
e_{m}
\end{array}\right], \quad \bar{f}=\left[\begin{array}{c}
f_{1} \\
f_{2} \\
\vdots \\
f_{m}
\end{array}\right] .
$$

Matrices $p$ and $q$ have the same size and structure as $e$, that is constant rows. These matrices generate strikingly simple results, and have many pleasing properties, for example the orthogonality conditions hold:

$$
e^{\prime}(B A-I)=0, \quad p^{\prime}\left(B^{-1}-A\right)=0 .
$$

Example 1 Here $|M|=-1$, D unit diagonal:

$$
M^{-1}=\left[\begin{array}{rrrrrrrr}
1 & 0 & 0 & 0 & 0 & 1 & 2 & -1 \\
0 & 1 & 0 & 0 & 0 & 1 & 2 & -1 \\
0 & 0 & 1 & 0 & 0 & 1 & 2 & -1 \\
0 & 0 & 0 & 1 & 0 & 1 & 2 & -1 \\
0 & 0 & 0 & 0 & 1 & 1 & 2 & -1 \\
1 & 1 & 1 & 1 & 1 & 1 & 1 & 1 \\
2 & 2 & 2 & 2 & 2 & 1 & 0 & 0 \\
-2 & -2 & -2 & -2 & -2 & 0 & 2 & 3
\end{array}\right]^{-1}
$$




$$
=\left[\begin{array}{rrrrrrrr}
1 & 0 & 0 & 0 & 0 & -8 & 7 & 3 \\
0 & 1 & 0 & 0 & 0 & -8 & 7 & 3 \\
0 & 0 & 1 & 0 & 0 & -8 & 7 & 3 \\
0 & 0 & 0 & 1 & 0 & -8 & 7 & 3 \\
0 & 0 & 0 & 0 & 1 & -8 & 7 & 3 \\
-2 & -2 & -2 & -2 & -2 & 80 & -69 & -30 \\
1 & 1 & 1 & 1 & 1 & -37 & 32 & 14 \\
0 & 0 & 0 & 0 & 0 & -2 & 2 & 1
\end{array}\right] .
$$

Irrespective of the size $n$ of matrix $M$, if (2) is satisfied, its inverse will be an arrow matrix. The size of $D^{-1}, p$ and $q$ will depend on $n$ but their elements will not change, and only the elements of $B$ will depend on $n$.

\section{Characteristic Polynomial}

First, to find the determinant of $M$, we need a lemma. Note that in the remainder of this paper it is not necessary that (2) holds. 
Lemma 1 Define the matrices $Q_{n, m}$ and $Q$ as

$$
Q_{n-m, m}=\left[\begin{array}{lllll}
0 & & & & e^{\prime} \\
& d_{2} & & & \vdots \\
& & \ddots & & \\
f & & & d_{n-m} & \\
f & \ldots & & & A
\end{array}\right], \quad Q=\left[\begin{array}{cc}
0 & \bar{e}^{\prime} \\
f & A
\end{array}\right],
$$

and put $Q_{m+1, m}=Q$. Then

$$
\left|Q_{n, m}\right|=\prod_{i=2}^{n-m} d_{i}|Q|, n>m .
$$

Proof. Expand $\left|Q_{m+i, m}\right|$ along the row $i$.

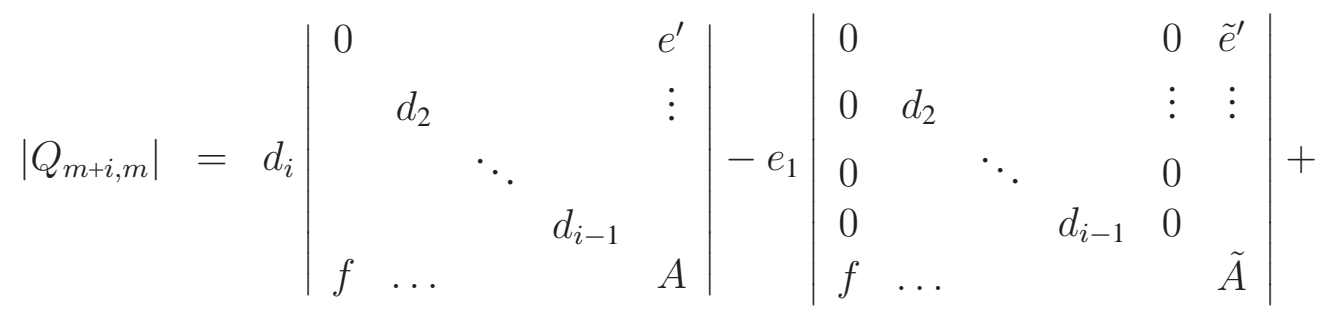




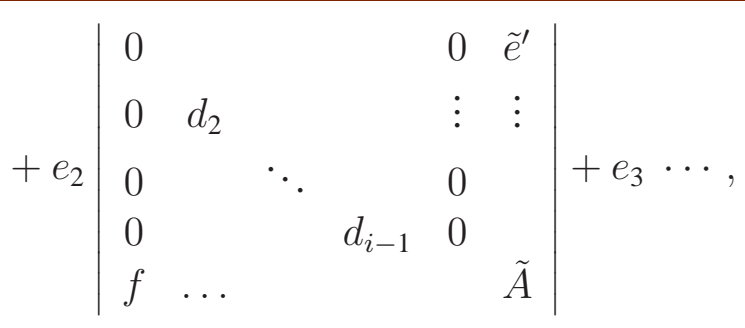

where $\tilde{A}$ indicates one column has been deleted from a matrix $A$.

Because $f$ has constant rows, all the determinants are zero except the first, giving $\left|Q_{m+i, m}\right|=d_{i}\left|Q_{m+i-1, m}\right|$, and the lemma follows.

Theorem 2 The determinant of the arrow matrix (1) is given by

$$
|M|=|A| \prod_{j=1}^{n-m} d_{j}+|Q| \sum_{i=1}^{n-m} \prod_{j=1, j \neq i}^{n-m} d_{j}, \quad n \geq m .
$$

If $D$ has a constant diagonal d, this simplifies to

$$
|M|=|A| d^{n-m}+|Q|(n-m) d^{n-m-1},
$$

with $Q$ as above. 
Proof. Expand determinant along top row:

$$
|M|=d_{1}\left|\begin{array}{ccccc}
d_{2} & & & & e^{\prime} \\
& d_{3} & & & \vdots \\
& & \ddots & & \\
& & & d_{n-m} & \\
f & \ldots & & & A
\end{array}\right|+\left|\begin{array}{lllll}
0 & & & & e^{\prime} \\
& d_{2} & & & \vdots \\
& & \ddots & & \\
& & & d_{n-m} & \\
f & \ldots & & & A
\end{array}\right| .
$$

Using the notation $F\left(n, m, d_{1}: d_{n-m}\right)=|M|$, then

$$
\begin{aligned}
|M| & =F\left(n, m, d_{1}: d_{n-m}\right) \\
& =d_{1} F\left(n-1, m, d_{2}: d_{n-m}\right)+Q_{n, m} \\
& =d_{1}\left(d_{2} F\left(n-2, m, d_{3}: d_{n-m}\right)+Q_{n-1, m}\right)+Q_{n, m} \\
& =\prod_{j=1}^{n-m} d_{j} F(m, m, 0)+\prod_{j=1}^{n-m-1} d_{j} Q_{m+1, m}+\cdots+d_{1} Q_{n-1, m}+Q_{n, m} .
\end{aligned}
$$

Applying the above lemma and the identity $F(m, m, 0)=|A|$ the result follows.

We can now write out the characteristic polynomial of $M$.

Theorem 3 The characteristic polynomial of the matrix $M$ defined as above is given by

$$
|A-\lambda I| \prod_{j=1}^{n-m}\left(d_{j}-\lambda\right)+\left|Q_{\lambda}\right| \sum_{i=1}^{n-m} \prod_{j=1, j \neq i}^{n-m}\left(d_{j}-\lambda\right)
$$


where

$$
Q_{\lambda}=\left[\begin{array}{cc}
0 & \bar{e}^{\prime} \\
\bar{f} & A-\lambda I
\end{array}\right] .
$$

If D has a constant diagonal d, the characteristic polynomial is

$$
|A-\lambda I|(d-\lambda)^{n-m}+\left|Q_{\lambda}\right|(n-m)(d-\lambda)^{n-m-1}, \quad n \geq m .
$$

For example, the eigenvalues of $M$ in Example 1 (for any $n$ ) are the roots of

$$
(1-\lambda)^{n-4}\left((1-\lambda)\left(-1-2 \lambda+4 \lambda^{2}-\lambda^{3}\right)+(n-3)\left(23 \lambda-7 \lambda^{2}\right)\right)=0
$$

with $n=8$ in this case. Numerically the roots are $-5.09395,0.00879162,1,1,1$, $1,3.2824,6.80275$.

\section{References}

[1] P. Concus, G. H. Golub, and G. Meurant, Block preconditioning for the conjugate gradient method, SIAM J. Sci. Stat. Comput. 6, (1985), 220-252.

[2] G. H. Golub and C. F. Van Loan, Matrix Computations, Second ed., (The Johns Hopkins University Press, Baltimore, MD, 1989).

[3] R. T. Gregory and D. L. Karney, A Collection of Matrices for Testing Computational Algorithms, (Wiley Interscience, New York, 1969). 
[4] M. Gu and S. C. Eisenstat, A divide-and-conquer algorithm for the symmetric tridiagonal eigenproblem, SIAM J. Matrix Anal. Appl. 16, (1995), 172-191.

[5] F. E. Hohn, Elementary Matrix Algebra. (The Macmillan Company, New York, 1958).

[6] J. K. Reid, Solution of linear systems of equations: direct methods, in Sparse Matrix Techniques, (ed. V.A.Barker), Lecture Notes in Mathematics 668 572, (Springer-Verlag, Berlin 1977), 109.

[7] O. Walter, L. S. Cederbaum, and J. Schirmer, The eigenvalue problem for 'arrow' matrices, J. Math. Phys. 25, (1984), 729-737.

[8] J. R. Westlake, A Handbook of Numerical Matrix Inversion and Solution of Linear Equations, (Wiley, New York, 1968).

[9] J. H. Wilkinson, The Algebraic Eigenvalue Problem, (Clarendon Press, Oxford, 1965), 95. 